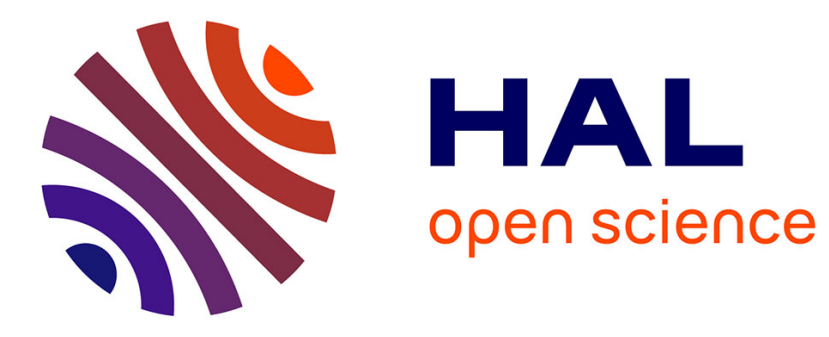

\title{
Benefit of the UltraZoom beamforming technology in noise in cochlear implant users
}

Isabelle Mosnier, Nathalie Mathias, Jonathan Flament, Dorith Amar, Amélie Liagre-Callies, Stéphanie Borel, Emmanuèle Ambert-Dahan, Olivier Sterkers, Daniele Bernardeschi

\section{To cite this version:}

Isabelle Mosnier, Nathalie Mathias, Jonathan Flament, Dorith Amar, Amélie Liagre-Callies, et al.. Benefit of the UltraZoom beamforming technology in noise in cochlear implant users. European Archives of Oto-Rhino-Laryngology, 2017, 10.1007/s00405-017-4651-3 . hal-01558185

\section{HAL Id: hal-01558185 \\ https://hal.sorbonne-universite.fr/hal-01558185}

Submitted on 11 Jul 2017

HAL is a multi-disciplinary open access archive for the deposit and dissemination of scientific research documents, whether they are published or not. The documents may come from teaching and research institutions in France or abroad, or from public or private research centers.
L'archive ouverte pluridisciplinaire HAL, est destinée au dépôt et à la diffusion de documents scientifiques de niveau recherche, publiés ou non, émanant des établissements d'enseignement et de recherche français ou étrangers, des laboratoires publics ou privés. 


\section{Benefit of the UltraZoom Beamforming}

\section{Technology in Noise in Cochlear Implant Users}

4 Isabelle Mosnier ${ }^{1,2}$, Nathalie Mathias ${ }^{3}$, Jonathan Flament ${ }^{2,4}$, Dorith Amar $^{2,5}$, Amelie Liagre ${ }^{2}$,

5 Stephanie Borel $^{2}$, Emmanuèle Ambert-Dahan ${ }^{2}$, Olivier Sterkers ${ }^{1,2}$ and Daniele Bernardeschi ${ }^{1,2}$

6

7 1. Sorbonne Universités, Université Pierre et Marie Curie Paris 6, Inserm, Unité Réhabilitation chirurgicale mini-invasive et robotisée de l'audition, Paris, France

9 2. AP-HP, Groupe Hospitalier Universitaire Pitié-Salpêtrière, Service ORL, Otologie, implants auditifs et chirurgie de la base du crâne, Paris, France

11 3. Advanced Bionics, Bron, France

12 4. Laboratoire AudioNova, Paris, France

13 5. Laboratoire Coscas Audition, Paris, France

14 Financial Disclosures/Conflict s of Interests: One of the authors (NM) is employee of

15 Advanced Bionics

16 Acknowledgment The authors gratefully acknowledge Solange Lator who collected the data.

17 Ethical Committee: Informed consent was obtained from each participating subject and ethics

18 approval was given by the French ethical committee: óComité de Protection des Personnes ï Ile-

19 de-France VIô Subjects obtained no reward or personal gain from taking part in the study. The

20 study was conducted in accordance with the Declaration of Helsinki and followed Good Clinical

21 Practice Guidelines.

22 Address correspondence to Isabelle Mosnier, GH Pitié-Salpétrière, Service ORL, 47-83

23 Boulevard de lấhôpital, 75651 Paris Cedex 13, France.

24 Tel +33 142162612. Fax. +33142162605. E-mail: isabelle.mosnier@aphp.fr 
26 Exchange of the sound processor of cochlear implants (CI) allows existing implant recipients to 27 take advantage of any advances in sound processor technology by exchanging or upgrading their

28 current processor to a newer model [1,2]. Funding of processor upgrade differs from one country 29 to another. Considering the high prices of the processors, the benefit provided by new processors 30 must be demonstrated.

31 In 2013, Advanced Bionics (AB, Stäfa, Switzerland) introduced the Naída CI Q70 (Naída CI) 32 sound processor. As well as being compatible with the newest $\mathrm{AB}$ cochlear implant systems, it 33 was also compatible with the existing HiRes $90 \mathrm{KE}$ and CIIE cochlear implant systems and 34 therefore existing recipients of $\mathrm{AB}$ devices, who were using older sound processor types, could be 35 upgraded to the newer technology. In addition to the functions and sound processing technology 36 already available in the previous generation sound processors, the Naída CI introduced an 37 acoustic signal processing beamforming technology called UltraZoom, which was already used in 38 Phonak hearing aids (Nyffeler, Reference Note 1). The intention was to help AB implant 39 recipients to communicate more easily and effectively in noisy environments, which still remains 40 a challenge, even for the best performing recipients [3].

41 UltraZoom is an adaptive multi-channel dual-microphone beamformer that focuses on input 42 originating from in front of the listener, while attenuating sounds coming from the sides and from 43 the rear (Fig. 1). It works by exploiting timing and phase differences in the signal arriving at two 44 spatially separated front and back omnidirectional microphones, positioned on top of the 45 processor. The inputs from the two microphones are subtracted from each other, after applying an 46 appropriate delay, and a front-facing directionality pattern is created, reducing input from the rear 47 hemisphere and creating a null point where sounds are completely attenuated. The adaptive nature 48 of UltraZoom allows it to constantly change the directionality of the null, based on the loudest 
49 noise source in 33 separate channels, thus suppressing moving noise sources as well as static ones 50 [4].

51 Previous studies evaluating adaptive beamforming technology with Cochlear Ltd. CI devices 52 have shown that it can significantly improve the perception of speech in noise [5-98]. Geißler et 53 al. [4] tested UltraZoom as implemented in the Naída CI in 10 subjects, and showed significant 54 improvement in speech perception in noise in a variety of challenging and realistic conditions, 55 when compared to the Harmony sound processor. However, subjects had no take-home 56 experience with the new sound processor and therefore it is not known if they would have been 57 able to transfer these gains shown in the laboratory, into the real world. This is a potential issue 58 for all beamforming technologies, as CI users report smaller subjective benefits than expected 59 from laboratory testing [5]. In part, this may be due to the fact that listeners often find themselves 60 in situations where speech and noise sources are not sufficiently spatially separated, particularly 61 in reverberant environments, which results in cancellation of the speech signal as well as the 62 noise and reduces the signal to noise advantages gained [910,1412]. In the previous studies where 63 subjective measures have been reported, two failed to show a significant improvement in 64 subjective performance with the beamforming technologies using the Speech Spatial Qualities 65 questionnaire, even though the objective results did show a significant benefit $[5,910]$. Only

66 Mosnier et al. [1] did show a significant improvement in performance in both objective and

67 subjective measures using the Abbreviated Profile of Hearing Aid Benefit (APHAB) [1213],

68 when subjects using Cochlear Ltd. devices were upgraded to the newer CP810 speech processor 69 with additional directionality.

71 The objectives of this study were to compare the performance of a group of existing AB cochlear 72 implant users, who were upgraded to the new Naída CI sound processor, in a test of speech 73 perception in noise with and without UltraZoom and to compare their subjective performance 
74 with their current sound processor, to their subjective performance after upgrading to the new

75 Naída CI sound processor.

\section{METHODS}

\section{$78 \quad$ Subjects}

79 From February to November 2015, 34 adult subjects aged between 21 and 89 years old (mean $8052.8 \pm 18.5$ ) were prospectively enrolled in a single tertiary referent referral center. Subjects were 81 required to have at least one CII/HiRes $90 \mathrm{~K}$ cochlear implant, a postlingual onset of severe-to82 profound hearing loss (Ó6 years of age) and French as their first language. The demographic data 83 of these subjects is presented in Table 1 . Nine subjects were unilaterally implanted, 11 bilaterally 84 implanted and 14 were bimodal users with a hearing aid on the contralateral ear. All subjects 85 were experienced CI users (5 to 14.7 years, mean $6.9 \pm 1.8$ ) who were due to get a processor 86 upgrade to the Naída CI as part of their routine clinical care. A repeated measures design was 87 used, where subjects acted as their own controls.

\section{$88 \quad$ Fitting}

89 At the baseline visit, subjects were fitted with a loaner sound processor for the purposes of 90 testing, identical to their current processor. This was to ensure that all microphones were new and 91 working optimally. It was programmed with their current clinical program, including the speech

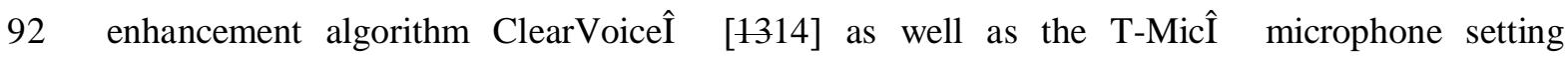
93 (microphone placed within the concha) [6,1415], if this was used on an everyday basis. They 94 were then upgraded to a new Naída CI sound processor, programmed with the same current 95 clinical program and an identical clinical program plus UltraZoom. The T-Mic microphone and 96 ClearVoice algorithm continued to be used with the Naída CI if they had been used with the 97 original processor. They were given a minimum of a two months take-home trial with the Naída

98 CI sound processor, where they were encouraged to use UltraZoom in appropriate situations, 
99 where speech was coming from the front and noise from the back and sides of the recipient. The

100 Advanced Bionics SoundWaveÊ programming software was used and all program parameters

101 remained the same, unless the subject was not happy with the sound quality, in which case

102 alterations to the current clinical program were made accordingly. All bilateral CI users except

103 one were upgraded on both sides.

104 Speech perception measures

105 Speech understanding in quiet was evaluated with two lists of seventeen monosyllabic words 106 each (Lafon lists) presented at $60 \mathrm{~dB}$ SPL from a source based at one meter in front of the subject.

107 Speech understanding in noise was measured with the Matrix sentence test in French [1516],

108 which is an adaptive test based on the Oldenburg sentence test (OlSa) [1617]. The subjects were

109 asked to repeat semantically unpredictable sentences, which always had the same structure:

110 Name, Verb, Number, Common name and Colour. A speech reception threshold (SRT) was

111 automatically measured by adjusting signal to noise ratio until a 50\% word understanding score

112 was reached. A lower SRT means a better performance. Prior to testing, at least two practice lists

113 (each containing 20 sentences) were presented to the subject to avoid training effects during the 114 test.

115 Sentences were presented from a loudspeaker located one meter in front of the subject (0-degree 116 azimuth). Non-correlated stationary speech shaped noise (SSN) was presented at a fixed level of

$11765 \mathrm{~dB}$ SPL simultaneously from all three loudspeakers positioned at $+/-90^{\circ}$ and $180^{\circ}$ to simulate

118 a diffuse noise environment. The level of the speech signal was varied to adjust the signal to noise

119 ratio. A low to moderately reverberant room was used, with a $\mathrm{T}_{60}$ of around 0.3 seconds.

120 Subjects were evaluated while listening with the technology that they utilized in their daily

121 environments; participants with a Naída CI processor on one ear and a contralateral hearing aid 122 were tested with both devices together, bilateral participants (two Naída CI processors or one 
123 Naída CI processor with another processor type contralaterally) were tested with both devices

124 turned on. The contralateral hearing aid was not fitted or changed during the follow-up period.

125 At the baseline visit, speech perception was measured with words in quiet with the current sound

126 processor and sentences in noise with the current sound processor and the new Naída CI sound

127 processor in the omnidirectional microphone mode, without UltraZoom. At the follow up visit,

128 two months later, speech perception was measured in quiet with the Naída CI processor without

129 UltraZoom and in noise with the Naída CI sound processor with and without UltraZoom (Table

130 2). The order of the speech test lists and the test conditions were randomized using a

131 randomization table prepared before the start of the study. At the end of the study the subjects

132 returned home with the new Naída CI sound processor.

\section{Subjective Testing}

134 Subjectôs self-assessment of their hearing with the different sound processors and programs was

135 recorded using the APHAB [1213]. This 24-item self-assessment inventory requires recipients to

136 report the amount of trouble they are having with communication in various everyday situations.

137 Benefit is calculated by comparing the recipient's reported difficulty in listening in the specified

138 scenarios. There are four subscales: Ease of Communication (EC), Reverberation (RV),

139 Background Noise (BN), and Aversiveness (AV). Scores are given on a scale from A to G where

140 A is ñI always experience thisò and G ñI never experience thisò. A percentage score from $1 \%$ to

$14199 \%$ is allocated to each category of response to give a mean percentage for each section. The

142 average score for each subsection, recorded at baseline with the previous sound processor, was

143 compared to the average score recorded at the two month follow up visit with the Naída CI sound

144 processor. A global score was also calculated, which is the mean of the scores for all the items in

145 the three EC, RV and BN subscales.

$146 \quad$ Statistics

147 The results for each test session were compared independently. Scores for words in quiet and the

148 Matrix sentence test in noise were not normally distributed, so a non-parametric Wilcoxon paired 
149 test were was used. Individual scores in quiet were compared using the binomial model described

150 by Thornton and Raffin [18]. The different subsections of the APHAB data were compared using

151 a series of non parametric Wilcoxon tests. A p value of less than 0.05 was considered to be

152 significant, A power calculation showed that in order to detect a difference of $2 \mathrm{~dB}$ with sufficient

153 power $(80 \%)$ and at a significance level of $\mathrm{p}=0.05$, a minimum of 17 subjects was required for the

154 objective testing.

\section{Speech perception testing}

159 When subjects were tested in quiet, there was no difference in group performance between the 160 previous sound processor(s) at baseline and the Naída CI processor(s) in omnidirectional mode

161 after two months use (median score of 53.8\% ranging from 5\% to $94 \%$ and median score of $16252.5 \%$ ranging from $0 \%$ to $97 \%$, respectively) (Wilcoxon paired test, $\mathrm{Z}=0.37, p>0.05$ ) (Fig. 21).

163 Analysis of individual scores using the binomial model, described by Thornton and Raffin 164 (1978), showed that four out of the 34 subjects had a significant improvement in scores between 165 the baseline and second test sessions (18). One subject saw a significant reduction in speech score 166 in quiet in the second test session, but scores in noise between sessions did not reflect this. All 167 other subjects had non significant differences between scores of less than $20 \%$ (Table 3 ).

168 Twenty-one out of the 34 subjects had sufficiently good performance in quiet with the previous 169 sound processor(s) at the initial session to be able to perform the Matrix test in noise (median 170 scores of $64 \%$ for monosyllabic words versus $23 \%$ in the group of 13 subjects who were not able 171 to perform test in noise). Matrix test at the follow-up session was only performed in this group of 17221 patients. At the initial baseline session, there was no significant difference between the 173 recipientsô previous sound processor(s) (median SRT of $-1.1 \mathrm{~dB}$ ) and the Naída CI sound 174 processor(s) (median SRT of $-1.2 \mathrm{~dB}$ ) for performance in noise when using the omnidirectional 
175 microphone (Wilcoxon paired test, $\mathrm{Z}=1.01, p>0.05$ ) (Fig. 3ZA, a lower SRT means a better 176 performance).

177 At the follow up session, after two months experience with the Naída CI sound processor(s), the 178 median SRT score with Naída CI with UltraZoom was $-4 \mathrm{~dB}$ (range: $+4.8 ;-10.5 \mathrm{~dB}$ ) compared to $179-0.45 \mathrm{~dB}$ (range: $+6.5 ;-8.0 \mathrm{~dB}$ ) with the Naída CI in omnidirectional mode (without Ultrazoom). 180 The use of UltraZoom significantly improved the median SRT by $3.6 \mathrm{~dB}$ (range: $+0.5 ;-7.8 \mathrm{~dB}$ )

181 (Wilcoxon paired test, Z=3.91, $p<0.0001$ ) (Fig. 3ZB).

182 Subjective evaluation

183 APHAB questionnaires were completed by all 34 subjects. When performance on the APHAB 184 questionnaire was compared across the sessions, significant differences between the scores with 185 the existing sound processor(s) at baseline and the Naída CI sound processor(s) for speech 186 understanding in noisy environments (Wilcoxon paired test, $\mathrm{Z}=3.57, p<0.001$ ), aversive situations 187 (Wilcoxon paired test, $\mathrm{Z}=2.10, p<0.05$ ) and globally (Wilcoxon paired test, $\mathrm{Z}=2.19, p<0.05$ ) were 188 obtained.

189 When looking at the APHAB outcomes for the group of 21 subjects who were able to perform the

190 Matrix test, a significant improvement when using the Naída CI sound processor(s) compared to 191 the previous processor(s) was found for speech understanding in noisy environments (Wilcoxon 192 paired test, $\mathrm{Z}=2.84, p<0.01$ ) and in aversive situations (Wilcoxon paired test, $\mathrm{Z}=2.10, p<0.05$ ) 193 (Fig. 43A). For the 13 subjects who were not able to perform the Matrix test at baseline, a 194 significant improvement when using the Naída CI sound processor(s) compared to the previous 195 processor(s) was also shown for speech understanding in noise (Wilcoxon paired test, $\mathrm{Z}=2.13$, $196 p<0.05)($ Fig. 43B). 
200 This study showed that for the 21 subjects who were able to complete the testing in difficult noisy 201 conditions, the use of UltraZoom provided a significant improvement in performance of $3.6 \mathrm{~dB}$ 202 SRT. The diffuse noise test conditions used in this study were designed to be challenging to 203 represent the most common noise condition that CI users encounter in everyday life. The addition 204 of some reverberation in the testing room also helped to simulate a real world condition and is 205 particularly relevant for beamforming technologies as when the target and interfering noise 206 become more spatially diffuse, beamforming performance can degrade [1011, 1112].

207 Our results were in line with the improvement seen by Geißler et al. [4] in a study evaluating 10 208 adult Harmony users who had been converted to the Naída CI, but had no take home experience 209 with the new processor. Subjects had been evaluated using the same adaptive test as in our study, 210 but in more challenging conditions with five loudspeakers used to create the noise environment 211 and a higher reverberation time of $0.6 \mathrm{~s}$. In our study, twenty-six out of the 34 subjects were using 212 the T-Mic in standard condition which already provides some directionality [6]. Some subjects 213 also used the ClearVoice static noise reduction technology, which in combination with 214 UltraZoom, has been shown to provide the greatest improvement in performance in noise [4, 215 1314]. We ehøose chose to keep the use of ClearVoice and/or the T-Mic constant across all test 216 conditions and for both sound processor types in order to have no impact on the results.

217 There is considerable variation in the degree of improvements reported for beamforming 218 technology. In previous studies, when compared to the omnidirectional microphone or the T-Mic, 219 UltraZoom improved speech reception thresholds in noise from 4 up to $9.8 \mathrm{~dB}$ in optimum 220 conditions [4,1314] (Advanced Bionics, Reference Note 2). Many factors can explain this 221 variation, such as the speech materials and noise type used, the configuration of the speaker array, 222 the microphone and program configurations compared. In addition, the head alignment of the 223 subject with the speech source can also affect the level of benefits of any adaptive beamformer. 224 Even though instructions about head position were provided to subjects prior to testing, this is 
225 something which remained difficult to control over the whole duration of the session. However,

226 these testing conditions reflected óeal lifeôconditions and show the wide range what level of

227 benefit a CI user could expect from using this new technology in their daily life. Unfortunately,

228 one of the limitations of adaptive SRT procedures is that a calculation of individual significant

229 SRT differences cannot be made based on the binomial model. The other limitation is that the

230 Matrix test was only performed in the group of the better performers, meaning that we cannot

231 ruled out that some poorer performers from the baseline session were finally able to do the test at

232 the follow-up session. Therefore, no information can be provided on the percentage of subjects

233 whose performance improved significantly when using the beamformer.

235 The purpose of providing beamforming to CI users is to improve their ability to communicate in 236 the everyday noisy environments we all encounter. Whilst many studies have shown the benefits 237 of this technology in a laboratory setting $[4,5,7,8]$, a subjective evaluation by subjects is required 238 to show that these benefits can be achieved in real world scenarios. Moreover, the upgrade 239 process was part of the routine clinical practice of the clinic, so both good and poor performers 240 were enrolled. As a result, almost forty percent of the subjects in our study who had poor speech 241 comprehension score in quiet were unable to do the Matrix test in difficult noisy environment 242 before upgrade, but may still have some subjective benefit of the speech processor upgrade. The 243 APHAB results shown here indicate a significant subjective improvement for the listening in 244 background noise, aversiveness and global sections when using the Naída CI sound processor. To 245 check whether the poorer performers benefited from the new sound processor, the APHAB 246 questionnaire was analysed for this particular population and still showed a significant benefit in 247 the background noise section. It is particularly interesting to observe this improvement in poorer 248 performers, for whom the objective improvement could not be shown through the Matrix test. It 249 highlights the importance of evaluating subjective feedback from CI recipients to assess their 250 level of comfort in everyday life. Some previous studies using different subjective measures have 
251 been unable to show a subjective benefit along side the laboratory benefits shown $[5,8]$. Only

252 Mosnier et al. [1] did show a significant benefit on the APHAB when subjects upgraded from the 253 older Cochlear Esprit 3G and Freedom sound processors to the newer CP810. This lack of strong

254 evidence $\theta$ f for any subjective benefits of beamforming is not just an issue for its use in cochlear 255 implants, but is also a criticism for its use with hearing aids [1719]. The subjective results are 256 limited by the fact that the APHAB in common with most of the subjective measurement tools 257 available, relies on asking subjects about predetermined situations, which may not be relevant or 258 equally important to all subjects. An additional limitation of any study where subjects are 259 upgraded to newer technology and cannot be blinded to the sound processor used is that 260 responses may be biased towards the newer technology.

262 The results of the speech perception testing at baseline show that group performance with the new

263 Naída CI sound processor in noise was the same as with the subjectsôexisting sound processor 264 when using the same programs and omnidirectional microphone settings. This provides clinicians 265 with confidence that subjects can be upgraded to the Naída CI without a change in performance 266 when used with the standard microphone settings and do not require any training period. 267 However, the subjects recruited were all using the Harmony sound processor, so these findings 268 can only be applied to recipients who are currently using this sound processor type.

269 The improvements in recipientsôuse of beamforming in real world environments may result from 270 a better understanding by clinicians of how to use the technology and appropriately counsel 271 recipients and better implementation of the beamforming algorithm, improving its robustness [9].

272 Indeed, appropriate counselling on the use of UltraZoom is crucial as recipients are required to 273 manually change the program depending on the listening situation encountered. Therefore, it is 274 important to provide recipients with concrete real life examples of situations where this feature 275 helps speech understanding. However, this might be less relevant with the newest generation of 
276 sound processors, which offers automatic selection of the microphone settings depending on the

277 incoming signal i.e. UltraZoom is switched on and off automatically depending on the 278 environment.

279 To conclude, this study showed that all subjects were successfully upgraded to the new Naída CI

280 Q70 sound processor. Once upgraded, subjects who were able to perform the French Matrix test

281 in noise with their previous processor could take advantage of the UltraZoom beamforming

282 technology on the new sound, so that their ability to communicate in noise was improved.

283 Ssubjective results with the APHAB questionnaire- confirmed these objective results, showing

284 improvements in median scores in the whole group, but also in the group of poorer performers,

285 for listening in background noise when using the Naída CI Q70 sound processor. This study

286 highlighted the importance of upgrading CI recipients to new technology and of including

287 adaptive tests in noise and subjective feedback evaluation as part of the process.

289 Author contribution statements: I.M. is the main researcher, designed experiments, analyzed

290 data and wrote the paper. J.F., D.A., A.L., S.B., E.A.D., M D. performed experiments. N.M.

291 provided writing assistance. D.B. and O.S. provided critical revision. All authors discussed the

292 results and implications and commented on the manuscript at all stages.

294 Compliance with Ethical Standards

295 Conflict of Interest: One of the authors (NM) is employee of Advanced Bionics. Other authors 296 declare they have no conflict of interest.

297 Ethical approval: All procedures performed in studies involving human participants were in 298 accordance with the ethical standards of the institutional and/or national research committee and 299 with the 1964 Helsinki declaration and its later amendments or comparable ethical standards.

300 Informed consent: Informed consent was obtained from all individual participants included in 301 the study. 
303

304 
306 1. Mosnier, I., Marx, M., Venail, F. et al. (2014) Benefits from upgrade to the CP810 sound

307 processor for Nucleus 24 cochlear implant recipients. Eur Arch Otorhinolaryngol. 271: 49-57. 308 doi: 10.1007/s00405-013-2381-8.

309 2. Seebens Y, Diller G (2012) Improvements in speech perception after the upgrade from the 310 TEMPO+ to the OPUS 2 audio processor. ORL J Otorhinolaryngol Relat Spec 74: 6-11. doi: $311 \quad 10.1159 / 000333124$.

312 3. Schafer EC, Pogue J, Milrany T (2012) List equivalency of the AzBio sentence test in noise 313 for listeners with normal-hearing sensitivity or cochlear implants. J Am Acad Audiol 23: 501314 509. doi: 10.3766/jaaa.23.7.2: 10.3766/jaaa.23.7.2.

315 4. Geißler G, Arweiler I, Hehrmann P et al. (2015) Speech reception threshold benefits in 316 cochlear implant users with an adaptive beamformer in real life situations. Cochlear Implants 317 Intern 16: 69-76. doi: 10.1179/1754762814Y.0000000088

318 5. Spriet A, Van Deun L, Eftaxiadis K et al. (2007) Speech understanding in background noise 319 with the two-microphone adaptive beamformer BEAM in the Nucleus Freedom Cochlear 320 Implant System. Ear Hear 28: 62-72. doi: 10.1097/01 aud.0000252470.54246.54

321 6. Gifford RH, Revit LJ (2010) Speech perception for adult cochlear implant recipients in a 322 realistic background noise: effectiveness of preprocessing strategies and external options for 323 improving speech recognition in noise. J Am Acad Audiol 21: 441-451. doi: $324 \quad$ 10.1097/01 aud.0000252470.54246.54

325 7. Brockmeyer AM, Potts LG (2011) Evaluation of different signal processing options in 326 unilateral and bilateral cochlear freedom implant recipients using R-Space background noise. $327 \quad$ J Am Acad Audiol 22: 65-80. doi: 10.3766/jaaa.22.2.2.

328 8. Hersbach AA, Arora K, Mauger SJ et al. (2012) Combining directional microphone and 329 single-channel noise reduction algorithms: a clinical evaluation in difficult listening 
conditions with cochlear implant users. Ear Hear 33: e13-e23. doi:

$331 \quad$ 10.1097/AUD.0b013e31824b9e21.

332 9. Buechner A, Dyballa KH, Hehrmann P, Fredelake S, Lenarz T.(2014) Advanced 333 beamformers for cochlear implant users: acute measurement of speech perception in 334 challenging listening conditions. PLoS One. Apr 22;9(4):e95542.

335 10. 9. Peterson PM, Wie SM, Rabinowitz WM et al. (1990) Robustness of an adaptive 336 beamforming method for hearing aids. Acta Otolaryngol Suppl, 469: 85-90.

337 11. 10. Greenberg JE, Zurek PM (1992) Evaluation of an adaptive beamforming method for 338 hearing aids. J Acoust Soc Am 91: 1662-1676.

339 12. 11. Kompis M, Dillier N (2001) Performance of an adaptive beamforming noise reduction 340 scheme for hearing aid applications. II. Experimental verification of the predictions. J Acoust $341 \quad$ Soc Am 109: 1134-1143.

342 13. 12. Cox RM, Alexander GC (1995) The abbreviated profile of hearing aid benefit. Ear Hear $343 \quad 16: 176-186$

344 14. 13. Büchner A, Brendel M, Saalfeld H, et al. (2010) Results of a pilot study with a signal 345 enhancement algorithm for HiRes120 cochlear implant users. Otol Neurotol 31: 1386ï 1390. 346 doi: 10.1097/MAO.0b013e3181f1cdc6.

347 15. 14. Kolberg E, Sheffield SW, Davis TJ et al. (2015) Cochlear implant microphone location 348 affects speech recognition in diffuse noise. J Am Acad Audiol 26: 51-58. doi: $349 \quad$ 10.3766/jaaa.26.1.6.

350 16. 15. Jansen S, Luts H, Wagener KC, et al. (2012) Comparison of three types of French speech351 in-noise tests: a multi-center study. Int J Audiol 51: 164-173. doi: $352 \quad 10.3109 / 14992027.2011 .633568$.

353 17. 16. Wagener KC, Kuehnel V, Kollmeier B (1999) Entwicklung und Evaluation eines 354 Satztests für die deutsche Sprache I: Design des Oldenburger Satztests. Zeitschrift für $355 \quad$ Audiologie 38: 44-56. 
356 18. Thornton AR, Raffin MJ. (1978) Speech-discrimination scores modeled as a binomial 357 variable. J Speech Hear Res. 1978 Sep;21(3):507-18.

358 19. 17. McCreery RW, Venediktov RA, Coleman JJ et al. (2012) An evidence-based systematic 359 review of directional microphones and digital noise reduction hearing aids in school-age 360 children with hearing loss. Am J Audiol 21: 295-312. doi: 10.1044/1059-0889(2012/12$361 \quad$ 0014).

\section{Reference Notes}

363 1. Nyffeler M (2010) StereoZoom, Improvements with directional microphones. Field study $364 \quad$ News Phonak AG, Switzerland

365 2. Advanced Bionics (2015) Advanced Bionics technologies for understanding speech in noise. 366 White paper. 
369 Fig. 1: Polar plot showing UltraZoom performance on KEMAR left ear.

370 Fig. 24: Performance score in quiet with the subjectô original sound processor(s) at 371 baseline and with the Naída CI sound processor(s) in omnidirectional mode at the follow-up

372 visit. Results are expressed as percentage of words correct for the lists of monosyllabic words in 373 quiet for the 34 subjects. The box plots show the first and third quartile values and the central 374 square, the median value. The whiskers indicate the non-outliers values for each group.

375 Fig. 32: Performance in noise for the 21 subjects who were able to perform MATRIX test in noise. A. At baseline with the subjectô original sound processor(s) and with the Naída CI

377 sound processor(s) in omnidirectional mode (without UltraZoom); B. At the follow-up visit with the Naída CI sound processor(s) in omnidirectional mode and with UltraZoom. Results are expressed as the speech reception thresholds (SRT, dB) for the Matrix sentence test in noise. A lower SRT means a better performance. The box plots show the first and third quartile

381 values and the central square, the median value. The whiskers indicate the non-outliers values for 382 each group. The asterisks indicate a statistically significant difference in performance (*** $383 p<0.0001)$

384 Fig. 43: Median scores for the APHAB self-assessment questionnaire at baseline with the 385 subjectô original sound processor(s) and at the follow-up visit with the Naída CI sound 386 processor(s). A. For the 21 subjects who were able to perform MATRIX test in noise; B. For 387 the 13 subjects who were not able to perform MATRIX test in noise

388 Scores are given for each of the four sub sections and a global value for the average of the Ease of 389 Communication, Reverberation and Background Noise sections. The box plots show the first and 390 third quartile values and the central square, the median value. The whiskers indicate the non391 outliers values for each subscale and each group. The asterisks indicate a statistically significant 392 difference in performance $(* p<0.05, * * p<0.01)$ 
Table 1 : Demographic details of the population

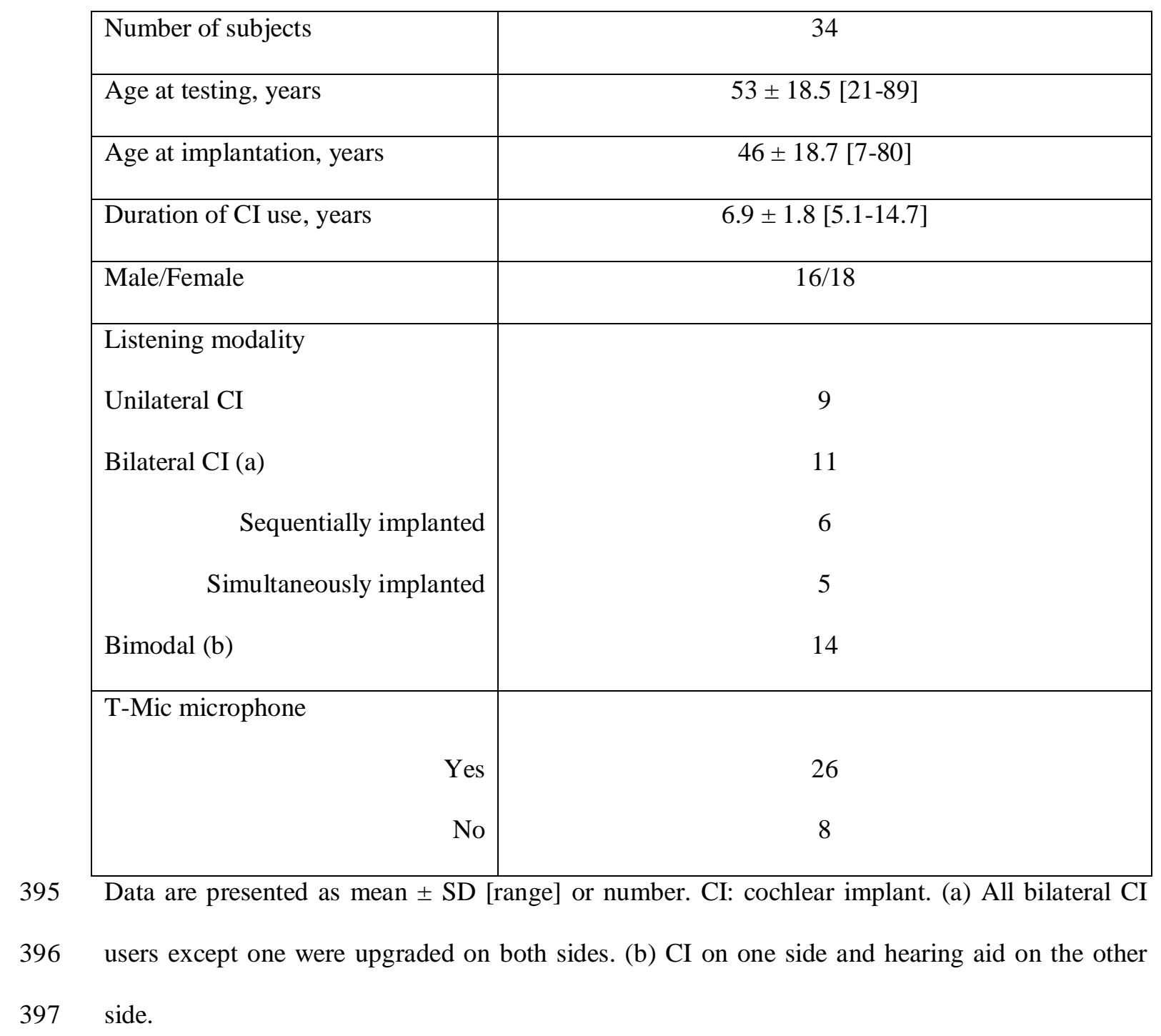


Table 2: Tests conducted at each visit.

400

\begin{tabular}{|c|c|}
\hline Baseline & $\begin{array}{l}\text { - Lists of words in quiet with the previous processor (s) } \\
\text { - } \quad \text { Speech test in noise with signal coming from the front } \\
\text { with the previous processor(s) and the Naída CI } \\
\text { processor (s) without UltraZoom in a random order } \\
\text { - APHAB questionnaire completed with regards to the } \\
\text { previous processor(s) use }\end{array}$ \\
\hline $\begin{array}{l}\text { Follow-up at } 2 \text { months after Naída } \\
\text { CI upgrade }\end{array}$ & $\begin{array}{l}\text { - Lists of words in quiet with the Naída CI processor(s) } \\
\text { without UltraZoom } \\
\text { - Speech test in noise with signal coming from the front with } \\
\text { the Naída CI processor(s) with and without UltraZoom in a } \\
\text { random order } \\
\text { - APHAB questionnaire completed with regards to the Naída } \\
\text { CI processor(s) use }\end{array}$ \\
\hline
\end{tabular}

401

402 
403

404

405

406

407

408

409

Table 3. Individual scores for speech perception testing in quiet for Lafon words at baseline with the previous sound processor and at the follow-up visit with the Naída CI processor in omnidirectional microphone mode.

\begin{tabular}{|cccccc|}
\hline $\begin{array}{c}\text { Subject } \\
\text { ID }\end{array}$ & $\begin{array}{c}\text { Lafon Words Score (\%) } \\
\text { Previous }\end{array}$ & Naida Omni & $\begin{array}{c}\text { Lubject } \\
\text { Processor }\end{array}$ & $\begin{array}{l}\text { Lafon Words Score (\%) } \\
\text { Previous } \\
\text { Processor }\end{array}$ & Naida Omni \\
\hline 1 & 37.5 & 58.5 & 18 & 52 & 55 \\
2 & 73 & 52.5 & 19 & 17 & 29 \\
3 & 67 & 61 & 20 & 76 & 58.5 \\
4 & 76 & 67 & 21 & 55.5 & 82 \\
5 & 35 & 17 & 22 & 17 & 41 \\
6 & 23 & 20 & 23 & 85 & 82 \\
7 & 26 & 43.5 & 24 & 14 & 23 \\
8 & 61 & 49.5 & 25 & 5 & 0 \\
9 & 35 & 38 & 26 & 58.5 & 11 \\
10 & 79 & 73 & 27 & 23 & 14 \\
11 & 94 & 94 & 28 & 46.5 & 52.5 \\
12 & 58 & 58.5 & 29 & 11 & 17 \\
13 & 64 & 52 & 30 & 64 & 79 \\
14 & 38 & 46.5 & 31 & 61 & 70 \\
15 & 29 & 32 & 32 & 82 & 73 \\
16 & 32 & 58 & 33 & 35 & 35 \\
17 & 64 & 67 & 34 & 94 & 97 \\
\hline
\end{tabular}


Figure 1

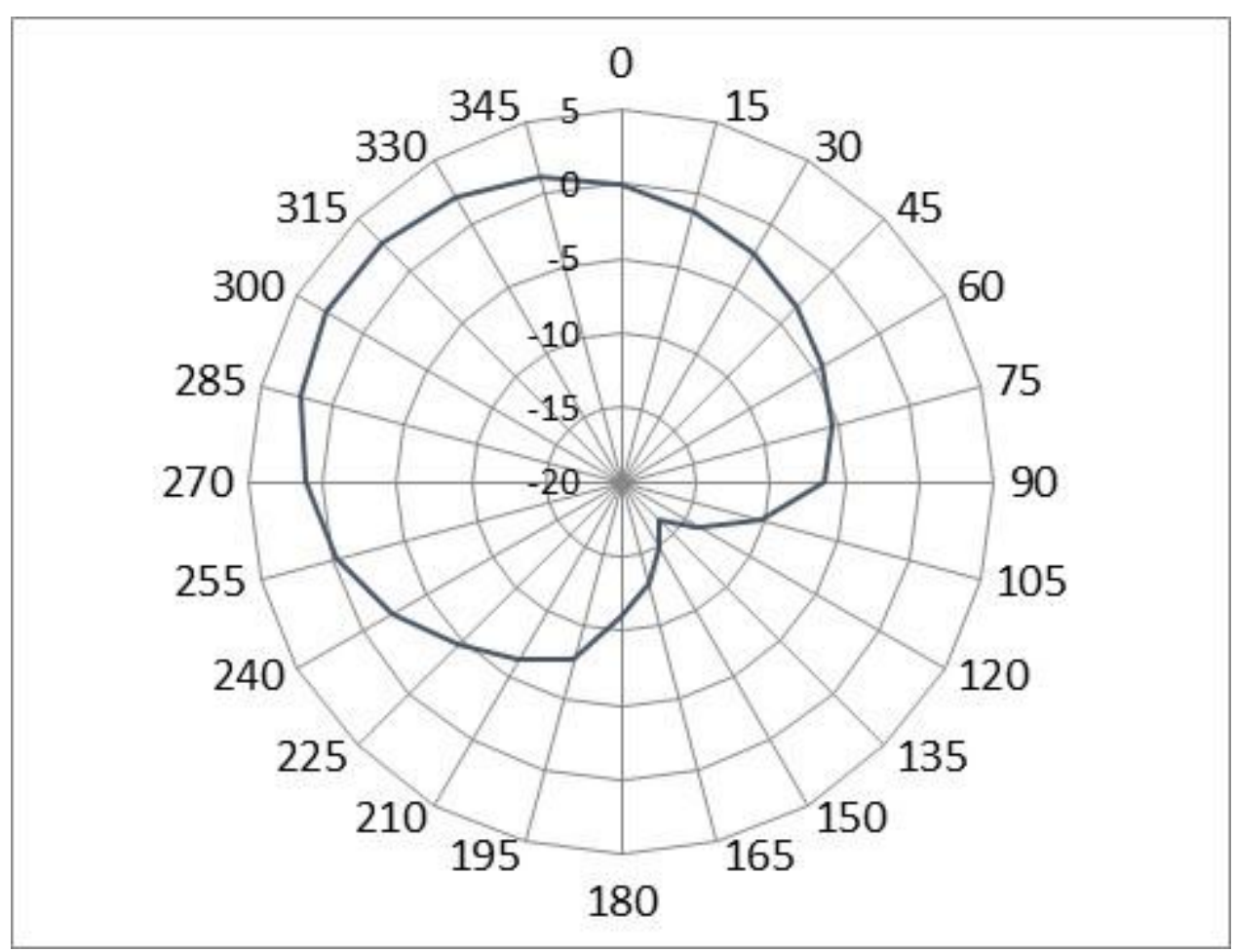


Figure 2

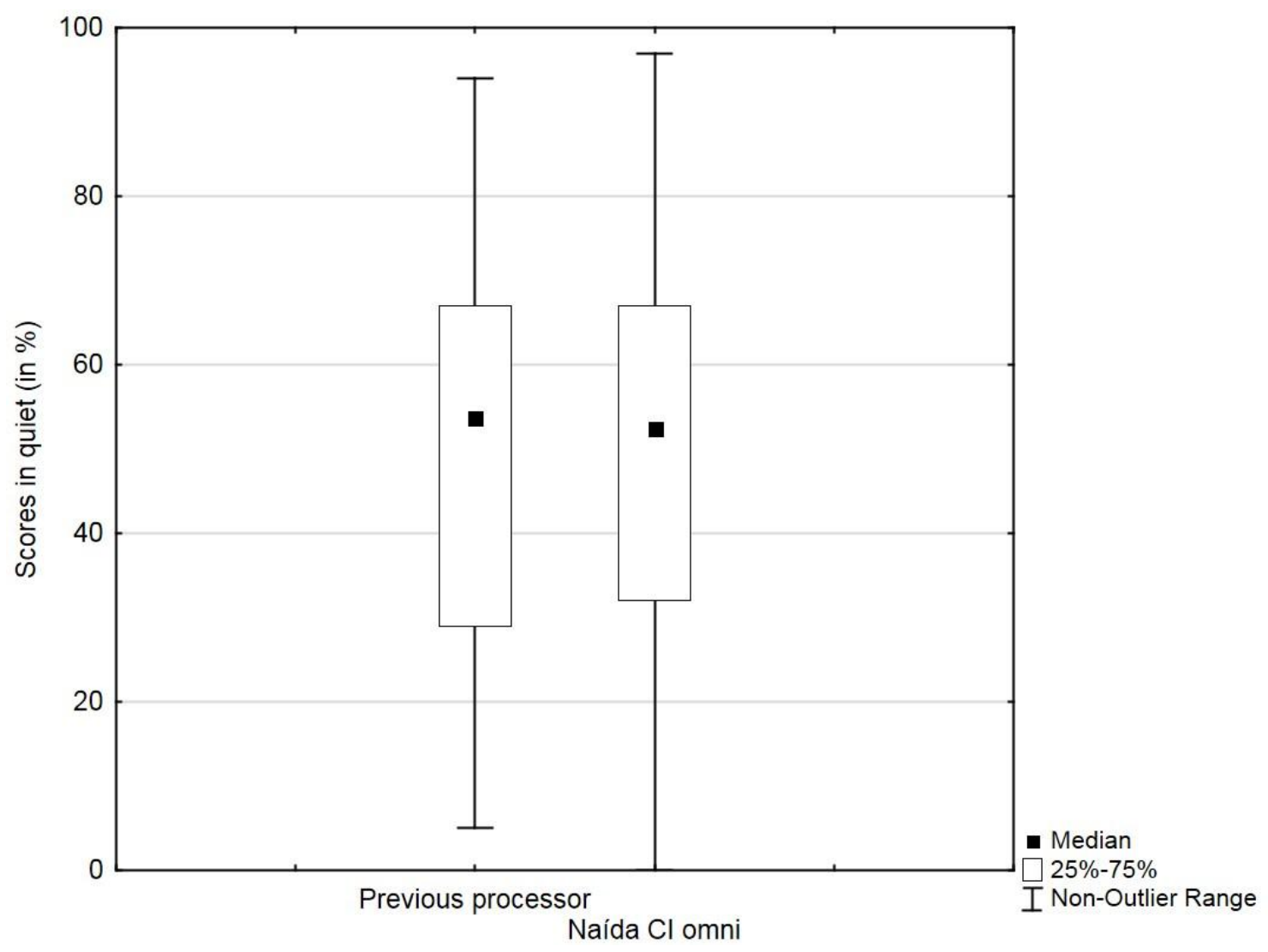


Figure $3 \mathrm{~A}$

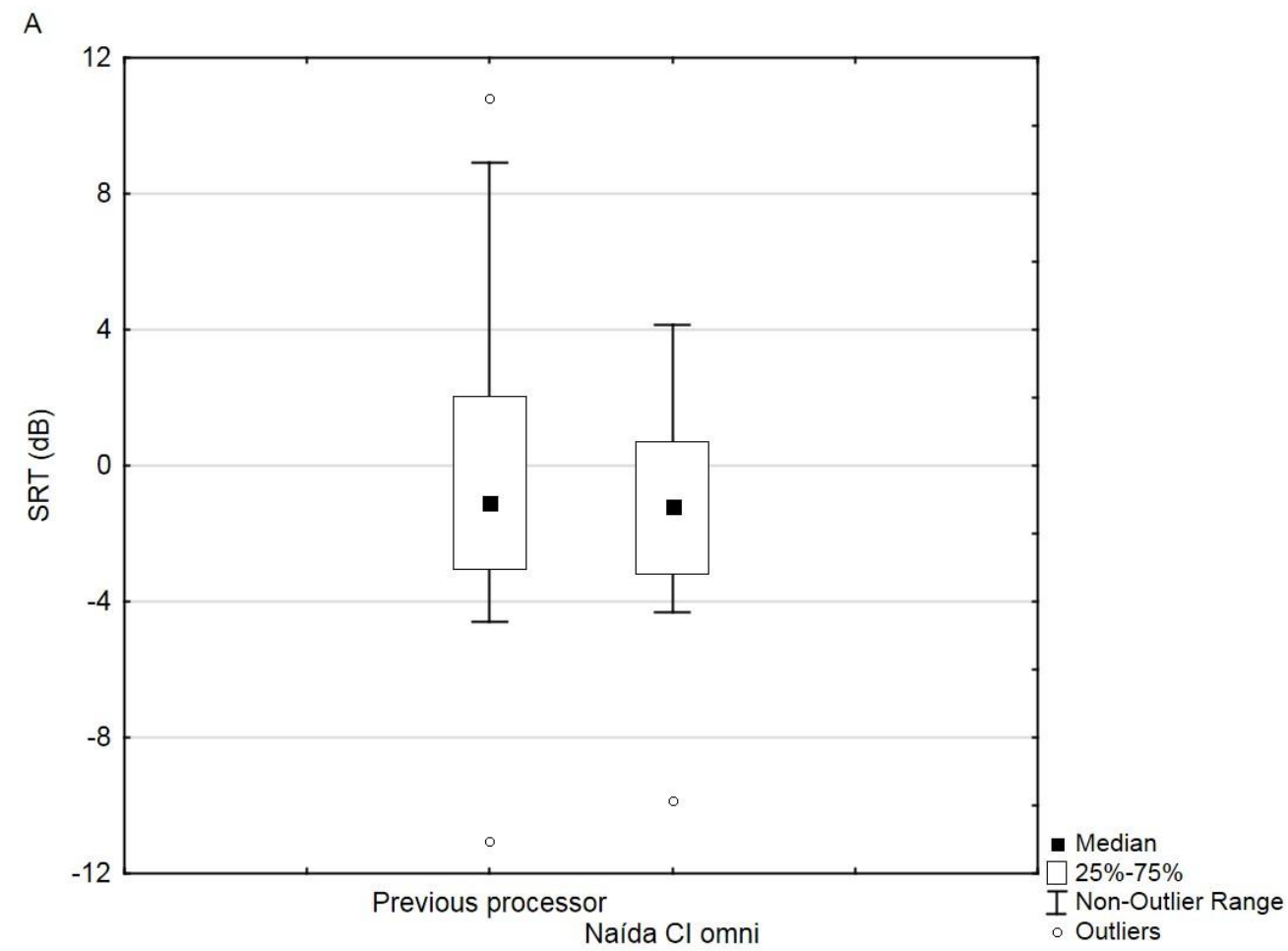


Figure 3B

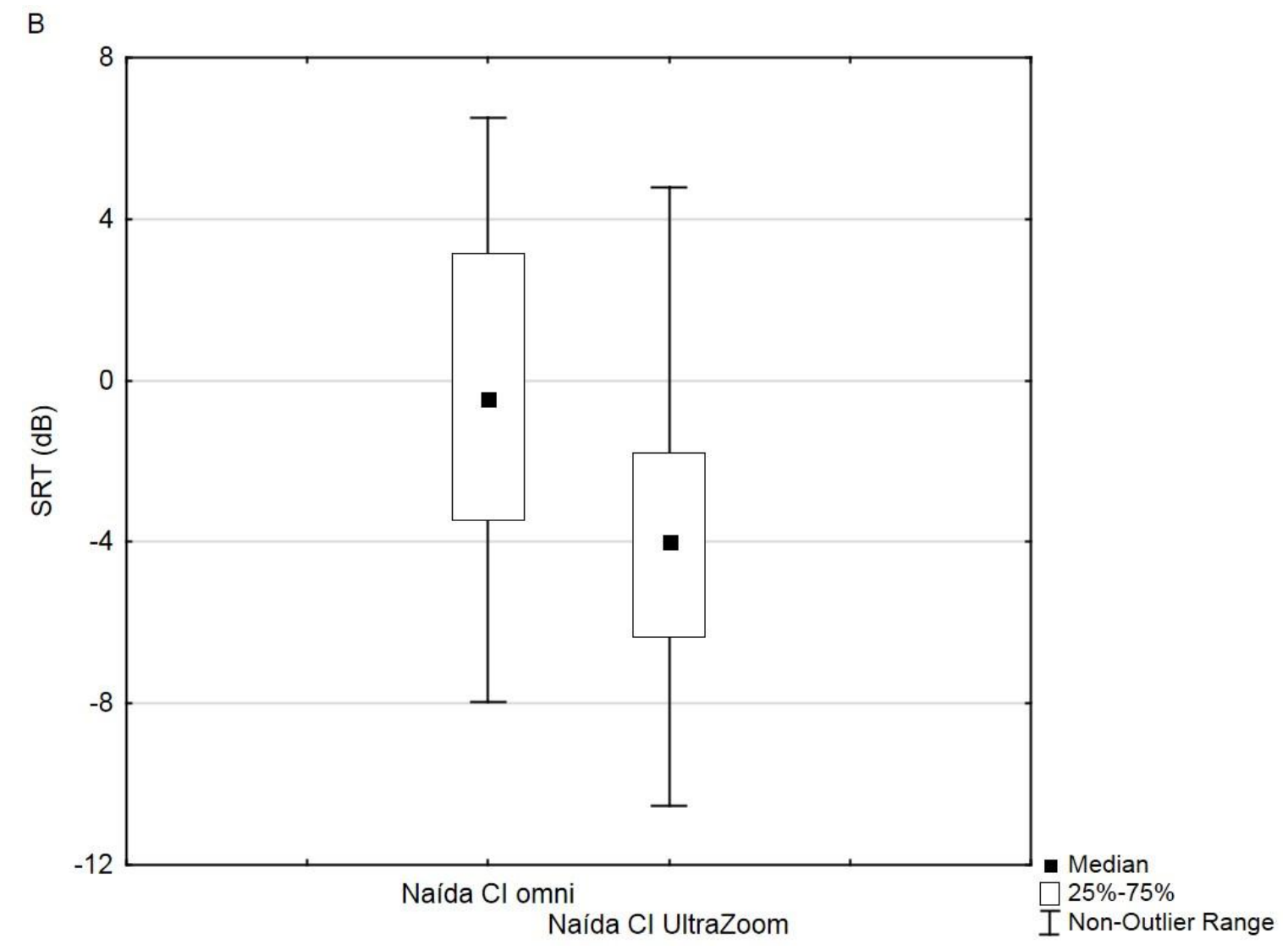




\section{Figure4A}

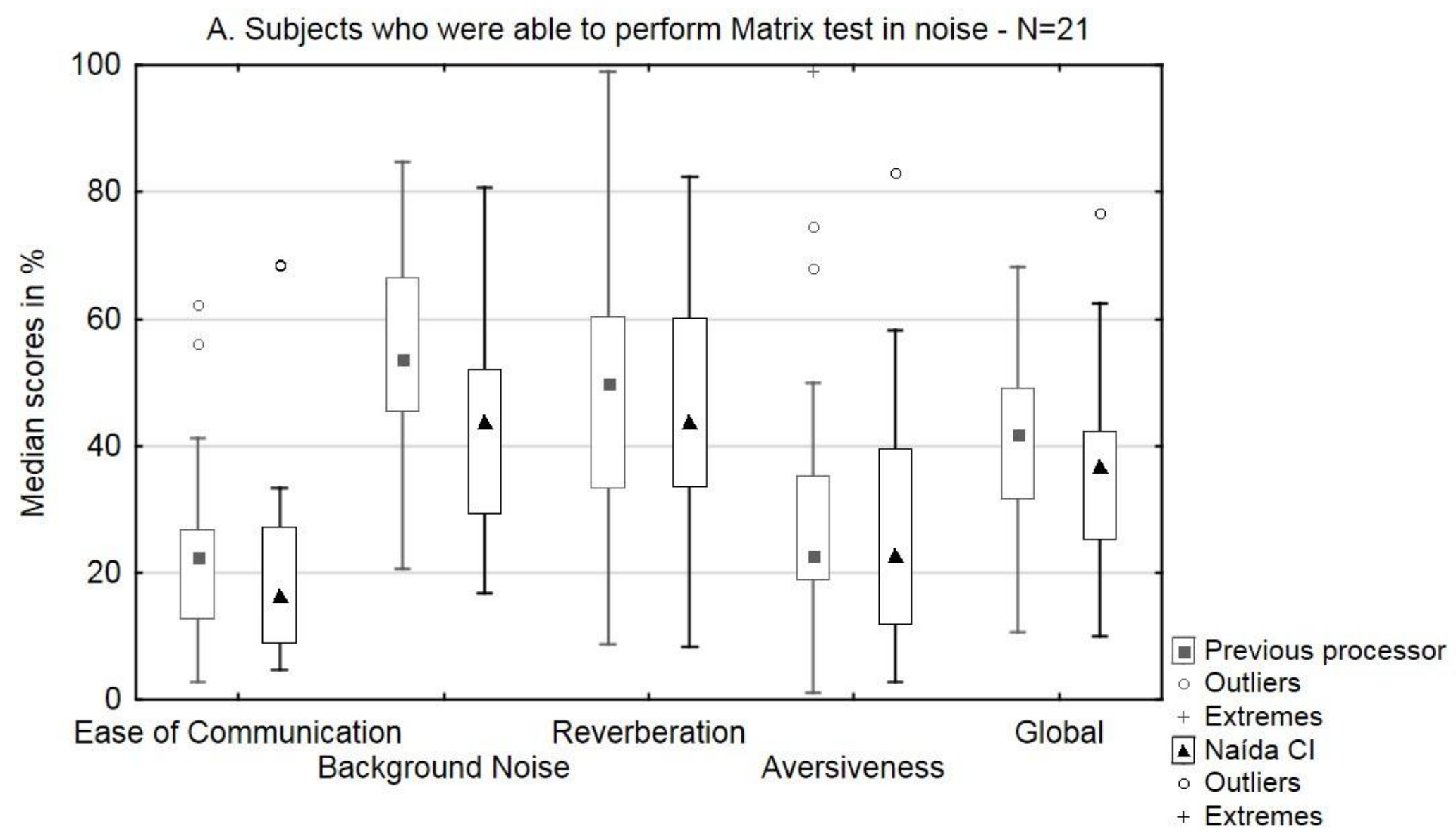


Figure 4B

* 